\title{
Moment Identities for Skorohod Integrals on Guichardet-Fock Spaces
}

\author{
Jihong Zhang1, Yongjun Lí2, Xiaochun Sun² \\ ${ }^{1}$ School of Mathematics, Lanzhou City University, Lanzhou, China \\ ${ }^{2}$ College of Mathematics and Statistics, Northwest Normal University, Lanzhou, China \\ Email: zhjhzhangjihong@163.com
}

Received 22 May 2016; accepted 16 July 2016; published 19 July 2016

\begin{abstract}
In this paper, we define expectation of $f \in F$, i.e. $E(f)=f(\varnothing)$, accordingto Wiener-Ito-Segal isomorphic relation between Guichardet-Fock space $F$ and Wienerspace $W$. Meanwhile, we prove a moment identity for the Skorohod integrals aboutvacuum state.
\end{abstract}

Keywords

Moment Identities, Skorohod Integral, Guichardet-Fock Spaces

\section{Introduction}

The quantum stochastic calculus [1] [2] developed by Hudson and Parthasarathy is essentially a noncommutative extension of classical Ito stochastic calculus. In this theory, annihilation, creation, and number operator processes in boson Fock space play the role of "quantum noises", [3] which are in continuous time. In 2002, Attal [4] discussed and extended quantum stochastic calculus by means of the Skorohod integral of anticipation processes and the related gradient operator on Guichardet-Fock spaces. Usually, Fock spaces as the models of the Particle Systems are widely used in quantumphysics. Meanwhile, vacuum states described by empty set on Guichardet-Fockspaces play very important role at quantum physics.

Recently Privault [5] [6] developed a Malliavin-type theory of stochastic calculus on Wiener spaces and showed its several interesting applications. In his article, Privault surveyed the moment identities for Skorohod integral on Wiener spaces. It is well known that Guichardet-Fock space F and Wiener space W are WienerIto-Segal isomorphic. Motivated by the above, we would like to study the momentidentities for Skorohod integraon Guichardet-Fock spaces.

This paper is organized as follows. Section 2, we fix some necessarynotations and recall main notions and facts about Skorohod integralin Guichardet-Fock spaces. Section 3 states our main results.

\section{Notations}

In this section, we fix some necessary notations and recall mainnotions in Guichardet-Fock spaces. For detail formulation of Skorohod integrals, we refer reader to [4].

Let $R_{+}$be the set of all nonnegative real numbers and $\Gamma$ the finite power set of $R_{+}$, namely 


$$
\Gamma:=\left\{\sigma \mid \sigma \subset R_{+}, \#<\infty\right\},
$$

where denotes the cardinality of $\sigma$ as a set. Particularly, let $\varnothing \in \Gamma^{(0)}$ be an atom of measure 1. We denote by $L^{2}(\Gamma)$ the usual space of square integral real-valued functions on $\Gamma$.

Fixing a complex separable Hilbert space $\eta$, Guichardet-Fock space tensor product $\eta \otimes L^{2}(\Gamma)$, which we identify with the space of square-integrable functions $L^{2}(\Gamma ; \eta)$, and is denoted by $F$.

For a Hilbert space-valued map $x: \Gamma \times R_{+} \rightarrow \eta$, let

$$
\delta(x): \sigma \mapsto \sum_{s \in \sigma} x_{s}(\sigma \backslash s)
$$

denotes the Skorohod integral operator. For a vector space-valued map $f: \Gamma \rightarrow V$, let $\nabla f$ and $D f$ be the maps $\Gamma \times R_{+} \rightarrow V$ given by

$$
\nabla f(\omega, s)=f(\omega \bigcup s), D f(\omega, s)=\mathbf{1}_{\{\omega<s\}} f(\omega \bigcup s)
$$

respectively denote the stochastic gradient operator of $f$ and the adapted gradient operator of $f$. Moreover, we write $\operatorname{Dom} \nabla$ for the domain of the stochastic gradient as an unbounded Hilbert apace operator:

$$
\operatorname{Dom} \nabla:=\left\{f \in F: \nabla f \in L^{2}\left(\Gamma \times R_{+} ; \eta\right)\right\} .
$$

Definition 2.1 For the map $x: \Gamma \times R_{+} \rightarrow \eta$, the value of Skorohod integral $\delta(x)$ at empty set is called the expectation of $\delta(x)$ on Guichardet-Fock space and is denoted by $E(\delta(x))$ i.e. $E(\delta(x))=\delta(x)(\varnothing)$.

Lemma 2.1 Let $x$ be a map $\Gamma \times R_{+} \rightarrow \eta$, if $x$ is square integrable and the function $(\omega, s, t) \rightarrow\left\langle x_{s}(\omega \bigcup t), x_{t}(\omega \bigcup s)\right\rangle$ is integrable, then $x \in \operatorname{Dom} \delta$ and

$$
\|\delta(x)\|^{2}=\int\|x\|^{2} d s+\iiint\left\langle x_{s}(\omega \bigcup t), x_{t}(\omega \bigcup s)\right\rangle d \omega d t d s,
$$

we denote

$$
\begin{aligned}
\operatorname{trace}(D x)^{2} & =\left\langle\nabla x, \nabla^{*} x\right\rangle \\
& =\int_{0}^{\infty} \int_{0}^{\infty}\left\langle\nabla_{t} x_{s}, \nabla_{s} x_{t}\right\rangle d t d s \\
& =\int_{0}^{\infty} \int_{0}^{\infty}\left\langle x_{s}(\omega \cup t), x_{t}(\omega \cup s)\right\rangle d t d s .
\end{aligned}
$$

Lemma 2.2 Let $f \in F$ and let $x: \Gamma \times R_{+} \rightarrow \eta$ be Skorohod integrable, if the map

$$
(\omega, s) \mapsto\left\langle x_{s}(\omega), f(\omega \cup s)\right\rangle
$$

is integrable, then

$$
\langle\delta(x), f\rangle=\iint\left\langle x_{\omega}, \nabla_{s} f(\omega)\right\rangle d \omega d s .
$$

Lemma 2.3 Let $x: \Gamma \times R_{+} \rightarrow \eta$ be measurable. For a.a.t, we have

$$
D_{t} \delta(x)=\delta_{0}^{t}\left(D_{t} x\right)+P_{t} x_{t},
$$

where $P_{t} x_{t}=\mathbf{1}_{\Gamma_{t}} x_{t}, \Gamma_{t}:=\{\omega \in \Gamma: \omega \subset[0, t[\}$.

Proof In view of the identity

$$
\mathbf{1}_{\{\sigma<t\}} \delta(x)(\sigma \cup t)=\sum_{s \in \sigma} \mathbf{1}_{\{\sigma<t\}} \mathbf{1}_{[0, t[}(s) x_{s}((\sigma / s) \cup t)+\mathbf{1}_{\{\sigma<t\}} x_{t}(\sigma),
$$

we have

$$
D_{t} \delta(x)(\sigma)=\delta\left(\mathbf{1}_{[0, t[}(\cdot) D_{t} x\right)(\sigma)+P_{t} x_{t}(\sigma) .
$$

\section{Moment Identities for Skorohod Integrals}

Theorem 3.1 For any $n \geq 1$ and $x \in F$, we have

$$
E\left(\delta(x)^{n+1}\right)=\sum_{k=1}^{n} \frac{n !}{(n-k) !} E\left[\delta(x)^{n-k}\left(\left\langle(\nabla x)^{k-1} x, x\right\rangle+\operatorname{trace}(\nabla x)^{k+1}+\sum_{i=2}^{k} \frac{1}{i}\left\langle(\nabla x)^{k-i} x, \nabla \operatorname{trace}(\nabla x)^{i}\right\rangle\right)\right],
$$


where

$$
\operatorname{trace}(\nabla x)^{k+1}=\int_{0}^{\infty} \cdots \int_{0}^{\infty}\left\langle\nabla_{t_{k-1}}^{*} x_{t_{k}}, \nabla_{t_{k-2}} x_{t_{k-1}} \cdots \nabla_{t_{0}} x_{t_{1}} \nabla_{t_{k}} x_{t_{0}}\right\rangle d t_{0} \cdots d t_{k} .
$$

For $n=1$ the above identity coincides with (2.1).

We will need the following lemma.

Lemma 3.1 Let $n \geq 1$ and $x \in F$. Then for all $1 \leq k \leq n$ we have

$$
\begin{aligned}
& E\left(\delta(x)^{n-k}\left\langle(\nabla x)^{k-1} x, \nabla \delta(x)\right\rangle\right)(\varnothing)-(n-k)\left(\delta(x)^{n-k-1}\left\langle(\nabla x)^{k} x, \nabla \delta(x)\right\rangle\right) \\
& =E\left[\delta(x)^{n-k}\left(\left\langle(\nabla x)^{k-1} x, x\right\rangle+\operatorname{trace}(\nabla x)^{k+1}+\sum_{i=2}^{k} \frac{1}{i}\left\langle(\nabla x)^{k-i} x, \nabla \operatorname{trace}(\nabla x)^{i}\right\rangle\right)\right] .
\end{aligned}
$$

Proof Using relation (2.2), (2.3), we obtain

$$
\begin{aligned}
& \delta(x)^{n-k}\left\langle(\nabla x)^{k-1} x, \nabla \delta(x)\right\rangle=\delta(x)^{n-k}\left\langle(\nabla x)^{k-1} x, x+\delta\left(\nabla^{*} x\right)\right\rangle \\
& \left.=\delta(x)^{n-k}\left\langle(\nabla x)^{k-1} x, x\right\rangle\right)+\left(\delta(x)^{n-k}\left\langle(\nabla x)^{k-1} x, \delta\left(\nabla^{*} x\right)\right\rangle\right. \\
& \left.=\delta(x)^{n-k}\left\langle(\nabla x)^{k-1} x, x\right\rangle\right)+\left(\left\langle\left(\nabla^{*} x\right), \nabla\left(\delta(x)^{n-k}(\nabla x)^{k-1} x\right)\right\rangle\right. \\
& \left.=\delta(x)^{n-k}\left\langle(\nabla x)^{k-1} x, x\right\rangle\right)+\left(\delta(x)^{n-k}\left\langle\nabla^{*} x, \nabla\left((\nabla x)^{k-1} x\right)\right\rangle+\left\langle\nabla^{*} x,\left((\nabla x)^{k-1} x\right) \otimes \nabla\left(\delta(x)^{n-k}\right)\right\rangle\right. \\
& =\delta(x)^{n-k}\left(\left\langle(\nabla x)^{k-1} x, x\right\rangle+\left\langle\nabla^{*} x, \nabla\left((\nabla x)^{k-1} x\right)\right\rangle\right)+(n-k) \delta(x)^{n-k-1}\left\langle\nabla^{*} x,\left((\nabla x)^{k-1} x\right) \otimes \nabla \delta(x)\right\rangle \\
& =\delta(x)^{n-k}\left(\left\langle(\nabla x)^{k-1} x, x\right\rangle+\left\langle\nabla^{*} x, \nabla\left((\nabla x)^{k-1} x\right)\right\rangle\right)+(n-k) \delta(x)^{n-k-1}\left\langle(\nabla x)^{k} x, \nabla \delta(x)\right\rangle,
\end{aligned}
$$

and

$$
\begin{aligned}
& \left\langle\nabla^{*} x, \nabla\left((\nabla x)^{k-1} x\right)\right\rangle=\int_{0}^{\infty} \cdots \int_{0}^{\infty}\left\langle\nabla_{t_{k-1}}^{\dagger} x_{t_{k}}, \nabla_{t_{k}}\left(\nabla_{t_{k-2}} x_{t_{k-1}} \cdots \nabla_{t_{0}} x_{t_{1}} x_{t_{0}}\right)\right\rangle d t_{0} \cdots d t_{k} \\
& =\int_{0}^{\infty} \cdots \int_{0}^{\infty}\left\langle\nabla_{t_{k-1}}^{\dagger} x_{t_{k}}, \nabla_{t_{k-2}} x_{t_{k-1}} \cdots \nabla_{t_{0}} x_{t_{1}} \nabla_{t_{k}} x_{t_{0}}\right\rangle d t_{0} \cdots d t_{k} \\
& =\operatorname{trace}(\nabla x)^{k+1}+\sum_{i=0}^{k-2} \int_{0}^{\infty} \cdots \int_{0}^{\infty}\left\langle\nabla_{t_{k-1}}^{\dagger} x_{t_{k}}, \nabla_{t_{k-2}} x_{t_{k-1}} \cdots \nabla_{t_{0}} x_{t_{1}} \nabla_{t_{k}} x_{t_{0}}\right\rangle d t_{0} \cdots d t_{k} \\
& =\operatorname{trace}(\nabla x)^{k+1}+\sum_{i=0}^{k-2} \int_{0}^{\infty} \cdots \int_{0}^{\infty}\left\langle\nabla_{t_{k-1}}^{\dagger} x_{t_{k}}, \nabla_{t_{k}} x_{t_{k+1}} \cdots \nabla_{t_{i+1}} x_{t_{i+2}}\left(\nabla_{t_{i}} \nabla_{t_{k}} x_{t_{i+1}}\right) \nabla_{t_{i-1}} x_{t_{i}} \cdots \nabla_{t_{0}} x_{t_{1}} x_{t_{0}}\right\rangle d t_{0} \cdots d t_{k} \\
& =\operatorname{trace}(\nabla x)^{k+1}+\sum_{i=0}^{k-2} \frac{1}{k-i} \int_{0}^{\infty} \cdots \int_{0}^{\infty}\left\langle\nabla_{t_{i}}\left\langle\nabla_{t_{k-1}}^{\dagger} x_{t_{k}}, \nabla_{t_{k}} x_{t_{k+1}} \cdots \nabla_{t_{i+1}} x_{t_{i+2}} \nabla_{t_{k}} x_{t_{i+1}}\right\rangle, \nabla_{t_{i-1}} x_{t_{i}} \cdots \nabla_{t_{0}} x_{t_{1}} x_{t_{0}}\right\rangle d t_{0} \cdots d t_{k} \\
& =\operatorname{trace}(\nabla x)^{k+1}+\sum_{i=0}^{k-2} \frac{1}{k-i}\left\langle(\nabla x)^{i} x, \nabla \operatorname{trace}(\nabla x)^{k-i}\right\rangle,
\end{aligned}
$$

Proof of Theorem 3.1, We decompose

$$
\begin{aligned}
& E\left(\delta(x)^{n+1}\right)=E\left(\left\langle x, \nabla(\delta(x))^{n}\right\rangle\right)=E\left(n(\delta(x))^{n-1}\langle x, \nabla \delta(x)\rangle\right) \\
& =\sum_{k=1}^{n} \frac{n !}{(n-k) !} E\left[\delta(x)^{n-k}\left(\left\langle(\nabla x)^{k-1} x, \nabla \delta(x)\right\rangle-(n-k)(\delta(x))^{n-k-1}\left\langle(\nabla x)^{k-1} x, \nabla \delta(x)\right\rangle\right],\right.
\end{aligned}
$$

then we apply lemma 3.1, which yields (3.1).

\section{Acknowledgements}

The authors are extremely grateful to the referees for their valuable comments and suggestions on improvement of the first version of the present paper. The authors are supported by National Natural Science Foundation of China (No. 11261027 and No. 11461061), supported by scientific research projects in Colleges and Universities in Gansu province (No. 2015A-122) and supported by Doctoral research start-up fund project of Lanzhou City Universities (No. LZCU-BS2015-02) and SRPNWNU (No. NWNU-LKQW-14-2).

\section{References}

[1] Hudson, R.L. and Parthasarathy, K.R. (1984) Quantum Ito’s Formula and Stochastic Evolutions. Communications in 
Mathematical Physics, 3, 301-323. http://dx.doi.org/10.1007/BF01258530

[2] Meyer, P.A. (1993) Quantum Probability for Probabilists. Lecture Notes in Mathematics, Spring-Verlag, Berlin. http://dx.doi.org/10.1007/978-3-662-21558-6

[3] Wang, C.S., Lu, Y.C. and Chai, H.F. (2011) An Alternative Approach to Privault's Discrete-Time Chaotic Calculus. Journal of Mathematical Analysis and Applications, 2, 643-654. http://dx.doi.org/10.1016/j.jmaa.2010.08.021

[4] Attal, S. and Lindsay, J.M. (2004) Quantum Stochastic Calculus with Maximal Operator Domains. The Annals of Probability, 32, 488-529. http://dx.doi.org/10.1214/aop/1078415843

[5] Privault, N. (2009) Moment Identities for Skorohod Integrals on the Wiener Space and Applications. Electronic Communications in Probability, 14, 116-121. http://dx.doi.org/10.1214/ECP.v14-1450

[6] Privault, N. (2010) Random Hermite Polynomials and Girsanov Identities on the Wiener Space. Infinite Dimensional Analysis, 13, 663-675. http://dx.doi.org/10.1142/S0219025710004218

\section{Submit or recommend next manuscript to SCIRP and we will provide best service for you:}

Accepting pre-submission inquiries through Email, Facebook, LinkedIn, Twitter, etc.

A wide selection of journals (inclusive of 9 subjects, more than 200 journals)

Providing 24-hour high-quality service

User-friendly online submission system

Fair and swift peer-review system

Efficient typesetting and proofreading procedure

Display of the result of downloads and visits, as well as the number of cited articles

Maximum dissemination of your research work

Submit your manuscript at: http://papersubmission.scirp.org/ 